\title{
Image Recovery Using a New Nonlinear Adaptive Filter Based on Neural Networks
}

\author{
L. Corbalán, G. Osella Massa, C. Russo, L. Lanzarini, A. De Giusti \\ III-LIDI, Faculty of Computer Sciences, National University of La Plata, Argentina
}

\begin{abstract}
This work defines a new nonlinear adaptive filter based on a feed-forward neural network with the capacity of significantly reducing the additive noise of an image. Even though measurements have been carried out using $\mathrm{x}$-ray images with additive white Gaussian noise, it is possible to extend the results to other type of images. Comparisons have been carried out with the Weiner filter because it is the most effective option for reducing Gaussian noise. In most of the cases, image reconstruction using the proposed method has produced satisfactory results. Finally, some conclusions and future work lines are presented.
\end{abstract}

Keywords: noise reduction, filters, neural networks.

\section{Introduction}

Digital image recovery entails a process which is meant to recover the original image data considering the degradation (noise in the acquisition, transmission problems, etc.) that this image has undergone. Its use is of utmost importance for several disciplines such as: Medicine, Biology, Physics, and Engineering.

The term noise in digital images refers to any pixel value of an image which does not match the reality quite exactly. Image acquisition is generally corrupted (noise) by the electronic equipment used for its capture (quantification noise, fog effect, etc.) and by the distortions generated in the transmission (possible interferences or errors in the transmission of data bits) [5].

Noise suppression is important since previous analysis referring to pattern recognition and segmentation are based on intensity value handling.

If conventional solutions are analyzed, it can be noticed that there exist specific filters for each type of noise [2], [9]. For instance, in the "salt and pepper" noise, the mean filter is the mostly used [1], [3], [10]; in the case of the Gaussian noise, Weiner filter is used.

It is important to notice that the application of a low-pass filter, like Weiner, despite being an effective way of reducing Gaussian noise in an image, does not produce good results with impulsive noise. This leads to the analysis of the noisy signal and considering, in each case, the solution to apply [8].

On the other hand, adaptive solutions exist that try to solve this kind of problems [6], [7].

This paper presents a new nonlinear adaptive filter capable of deleting additive noise based on neural networks. Its adaptive capacity to the input signal allows the network's training from generic-noisy signals thus avoiding the analysis of the type of filter to use in each case.

This paper is organized as follows: Section 2 describes the way in which the filter is applied to a noisy image. The neural network training is described in Section 3. Section 4 describes the result. And, finally, Section 5 presents the details of the conclusion together with some future work lines.

\section{Nonlinear Filter Based on a Feed-forward Neural Network}

The filter proposed in this paper is based on a feed-forward neural network which, after being 
trained, has the capacity of indicating the correction to be carried out in each pixel. This means that image recovery is carried out adding to each pixel the value obtained as result by the neural network.

The network has three layers: input, hidden, and output. The first one is made up by 8 neurons, the second by 5 , and the third by a single neuron that indicates the correction to be carried out.

NI refers to the noisy image with $n \times m$ dimension to be recovered using the neural network.

In order to ease the computations corresponding to each pixel's environment, the following four additional matrices are used:

$$
\begin{aligned}
E & =\left(\begin{array}{ll}
1 & -1
\end{array}\right) \otimes N I, \\
S & =\left(\begin{array}{c}
1 \\
-1
\end{array}\right) \otimes N I, \\
S W & =\left(\begin{array}{cc}
-1 & 0 \\
0 & 1
\end{array}\right) \otimes N I, \\
N W & =\left(\begin{array}{cc}
0 & -1 \\
1 & 0
\end{array}\right) \otimes N I
\end{aligned}
$$

The symbol $\otimes$ represents the matrix convolution.

For each pixel $(i, j)$ of the NI image, the input to the network is an eight-component vector made up as follows:

$$
\text { input }(i, j)=\left(\begin{array}{c}
E(i, j-1) \\
E(i, j) \\
S(i-1, j) \\
S(i, j) \\
S W(i-1, j-1) \\
S W(i, j) \\
N W(i-1, j) \\
N W(i, j-1)
\end{array}\right)
$$

This input vector spreads along the network as usual. The activation function for those hidden layer neurons is:

$$
f^{h}(x)=\frac{2}{1+e^{-2 x}}-1
$$

For the single neuron of the output layer, a linear activation function is applied:

$$
f^{o}(x)=x
$$

The result of the network is directly added to the noisy image pixel value, thus achieving its recovery.
The pseudo code corresponding to the previously described process is as follows:

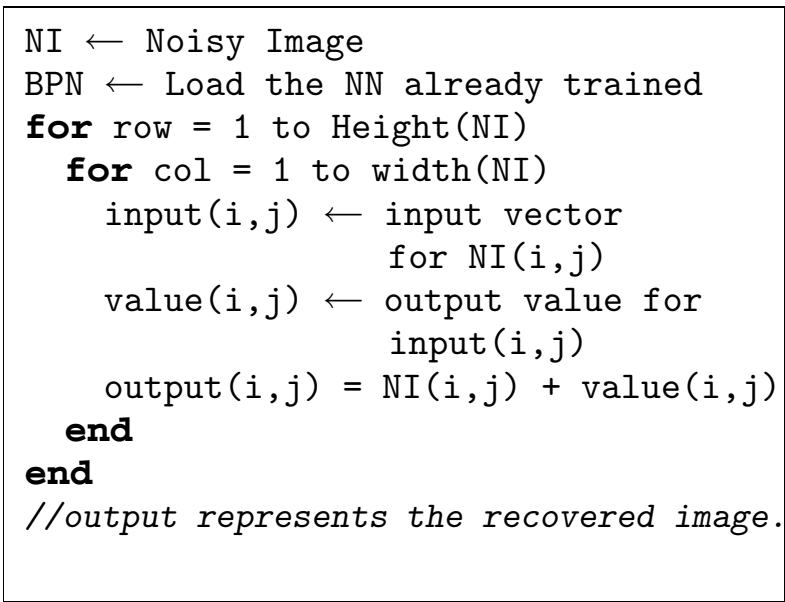

\section{Neural Network Training}

For its training the neural network uses the back propagation algorithm [4] taking as error measure the mean square error (MSE).

Since it is a supervised learning, test images to which additive noise has been applied have been used.

Each noise image pixel will be entered into the network as a vector - as indicated in the previous section - whose associated output value is the corresponding correction factor. Such factor is computed as follows:

$$
S(i, j)=I M(i, j)-N I(i, j)
$$

Notice that in this case the original image (IM) is available since the noise was introduced intentionally.

The selection of images used for training must assure the maximum coverage of all possible input patterns because the network is capable of interpolating missing information from these, but not extrapolating it. For this reason, one of the images used was Barbara because of its richness in different patterns.

\section{Results}

In order to train the neural network, images with Gaussian additive white noise were used. This type of noise is characterized by a continuum energy spectrum for all the frequencies. When this problem arises, the exact value of any pixel is different each time the same image is captured. 
This effect adds or subtracts a given value at a real gray level and is independent of the values taken by the image.

The information used in the network training must assure the representation of the different intensity combinations that may arise in a noisy image. For this reason, the images Lena and Barbara, both with size $512 \times 512$ pixels, were used.

Given that in the training stage those matrices of (1) are used and that they do not take into account the border values of the noisy image, the network input vectors are obtained from a $510 \times 510$ pixels region obtained by the original noisy image clipping.

The neural network was trained during 50 iterations with Barbara by means of the procedure described in Section 2. The Gaussian noise was applied with zero mean and 0.01 variance.

Table 1 shows the results obtained when applying the input neural network to a set of standard testing images, which are shown in Figure 1.

\begin{tabular}{|l||c|c|c|c|}
\multicolumn{1}{c||}{} & Noisy & Network & Wiener & Median \\
\hline \hline Barbara & 20.12 & $\mathbf{2 5 . 8 6}$ & 25.51 & 23.21 \\
\hline Lena & 20.06 & $\mathbf{2 7 . 3 7}$ & 27.20 & 26.78 \\
\hline Boat & 20.13 & $\mathbf{2 6 . 7 8}$ & 26.65 & 25.71 \\
\hline Peppers & 20.15 & 26.48 & $\mathbf{2 7 . 1 7}$ & 26.12 \\
\hline
\end{tabular}

Table 1. Results for each filter.

Each value in Table 1 is the best behavior of 20 different neural networks, each of which was obtained from an independent training. Figure 2 shows the results of applying different filters of Lena.

As it can be seen, the nonlinear adaptive filter based on neural networks proposed in this paper shows better results than the Weiner and Median filters in almost all the images. The shown numbers correspond to the peak signal-to-noise ratio defined in equation (4.1).

It is important to emphasize that the neural network has a general training and can be applied to any type of image with Gaussian noise. As counterpart, conventional methods should be selected according to the image intensity values and its distortion type. This leads us to obtain solutions that depend on the observer's appreciation.

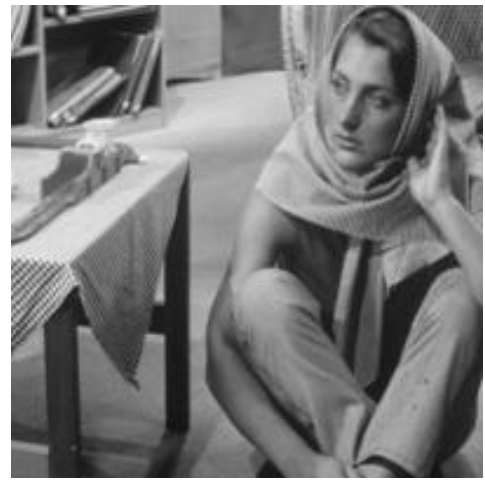

Barbara

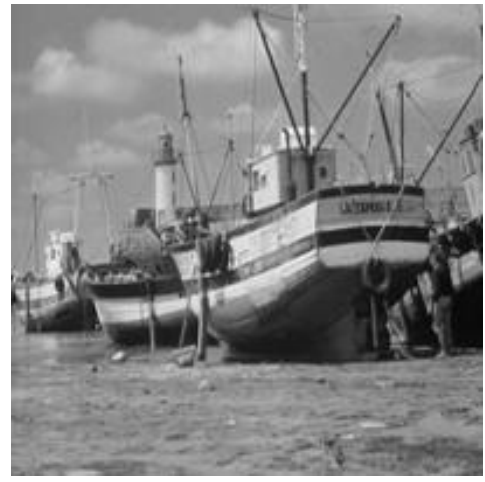

Boat

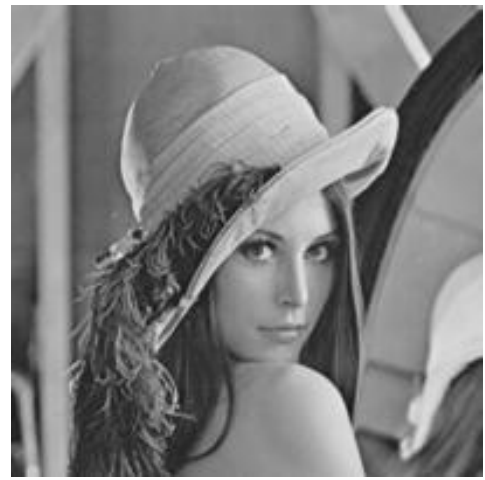

Lena

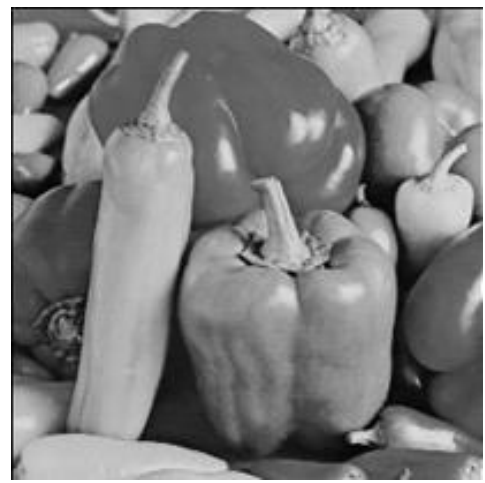

Peppers

Fig. 1. Test Images. 


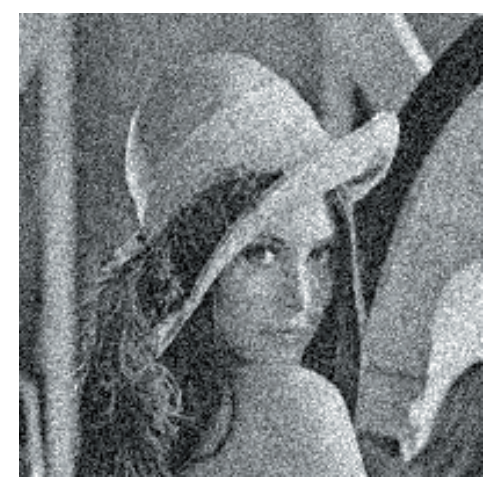

a) Noisy Image.

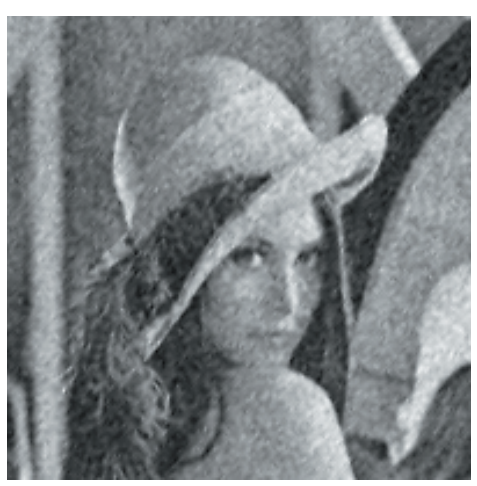

b) Filtered image using the network.

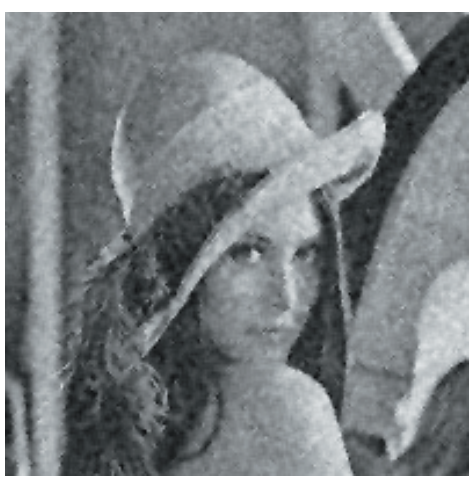

c) Filtered image using Wiener.

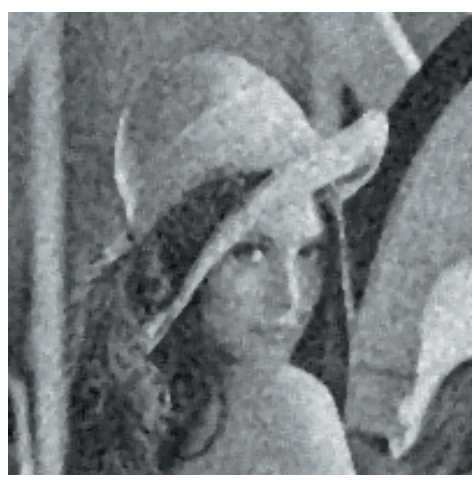

d) Filtered Image using Median.

Fig. 2. Results of applying different filters to the same noisy image.
On the other hand, the neural network allows implementing a more general filter, applicable to different situations. For instance, Table 2 shows the nonlinear adaptive filter based on the trained neural network with Barbara applied to the four X-ray images of Figure 3 to which Gaussian noise with zero mean and 0.005 variance was introduced. Table 3 shows the result of applying Gaussian noise with zero mean and 0.01 variance.

$$
P S N R=20 \log _{10}\left(\frac{1}{\sqrt{\frac{\sum_{i} \sum_{j}(I M(i, j)-N I(i, j))^{2}}{N}}}\right)
$$

\section{Conclusions}

A new nonlinear adaptive filter based on a feedforward neural network has been presented. It has shown satisfactory results in its application to images with additive noise.

Although this filter was proposed to be applied to $\mathrm{x}$-ray image with additive white Gaussian noise, experience shows good results when it is applied to other type of images.

It only remains to measure the neural network adaptive capacity to different parameters when generating the image with Gaussian noise.

We are currently working on the generalization of this filter application to other types of noises, apart from the Gaussian additive white noise.

\begin{tabular}{|l||c|c|c|c|}
\cline { 2 - 5 } \multicolumn{1}{c||}{} & Noisy & Network & Wiener & Median \\
\hline \hline Image 1 & 20.18 & $\mathbf{2 7 . 6 2}$ & 27.26 & 26.37 \\
\hline Image 2 & 20.04 & $\mathbf{2 7 . 9 4}$ & 26.23 & 27.54 \\
\hline Image 3 & 20.31 & $\mathbf{2 8 . 5 1}$ & 26.75 & 28.16 \\
\hline Image 4 & 20.24 & $\mathbf{2 6 . 9 7}$ & 26.18 & 26.52 \\
\hline
\end{tabular}

Table 2. Gaussian noise with 0.005 variance.

\begin{tabular}{|l|c|c|c|c|}
\cline { 2 - 5 } \multicolumn{1}{c|}{} & Noisy & Network & Wiener & Median \\
\hline \hline Image 1 & 20.36 & $\mathbf{2 8 . 0 5}$ & 27.96 & 27.62 \\
\hline Image 2 & 20.14 & $\mathbf{2 8 . 5 1}$ & 27.68 & 27.66 \\
\hline Image 3 & 20.45 & $\mathbf{2 6 . 4 2}$ & 26.34 & 25.42 \\
\hline Image 4 & 20.59 & $\mathbf{2 8 . 8 7}$ & 27.67 & 27.28 \\
\hline
\end{tabular}

Table 3. Gaussian noise with 0.01 variance. 


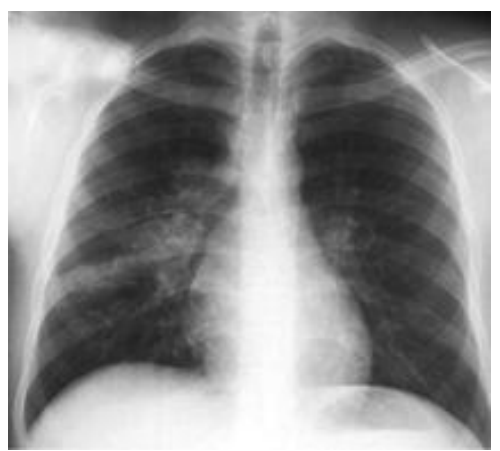

Image 1 .

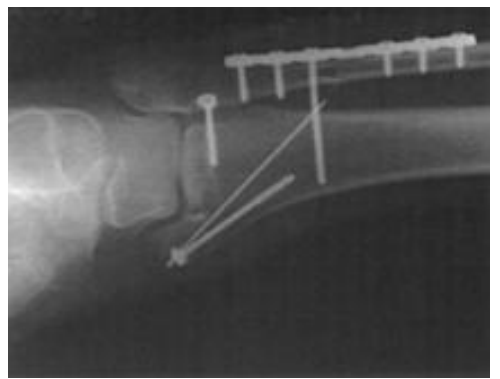

Image 2.

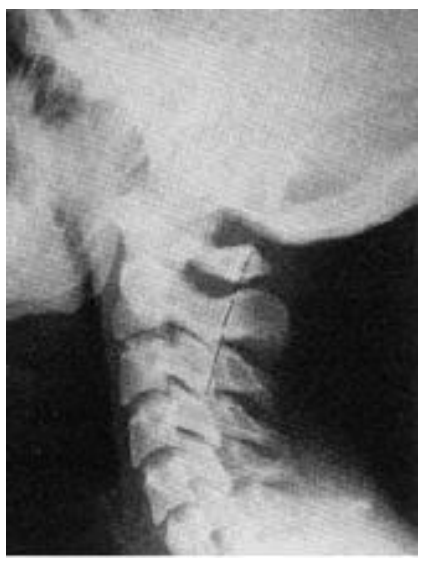

Image 3 .

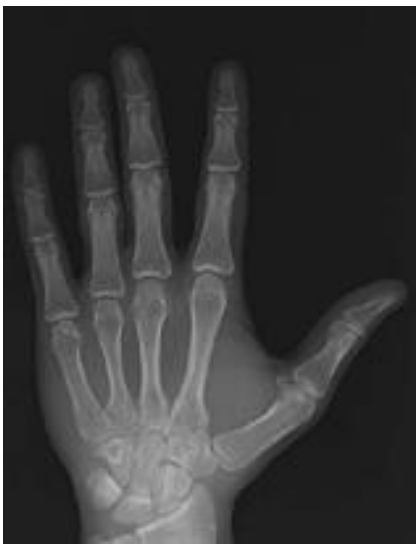

Image 4.

Fig. 3. X-ray test images.

\section{References}

[1] R. H. Chan, Chung-Wa Ho, M. Nikolova, Saltand-pepper noise removal by median-type noise detectors and detail-preserving regularization.; Image Processing, IEEE Transactions on Volume 14, Issue 10, Oct. 2005, pp. 1479-1485, DOI 10.1109/TIP. 2005.852196

[2] Chung-Chang Wu, Chun-Jun Huang, Jung-Hua WANG, Speckle noise reduction for ultrasonic images; Systems, Man and Cybernetics, 2003. IEEE International Conference on Volume 5, 5-8 Oct. 2003, pp. :4165-4170, Vol. 5.

[3] A. R. ForouZAN, B. N. ARAABI, Iterative median filtering for restoration of images with impulsive noise. Electronics, Circuits and Systems, 2003. ICECS 2003. Proceedings of the 2003 10th IEEE International Conference on Volume 1, 14-17 Dec. 2003, pp. 232-235, Vol. 1.

[4] A. Freeman James And M. Skapura DaVID, $R e$ des neuronales Algoritmos, aplicaciones y técnicas de programación, Ed. Addison-Wesley; 1993.

[5] C. F. GonzÁlez, R. E. Woods, Digital image processing, Addison Wesley; 2004.

[6] HAU-SAN WONG, LiNG GUAN, A neural learning approach for adaptive image restoration using a fuzzy model-based network architecture. Neural Networks, IEEE Transactions on Volume 12, Issue 3, May 2001, pp. 516-531, Digital Object Identifier $10.1109 / 72.925555$.

[7] E. S. Hore, B. QIU, H. R. WU, Adaptive noise detection for image restoration with a multiple window configuration. Image Processing. 2002. Proceedings. 2002 International Conference on Volume 1, 22-25 Sept. 2002 pp. I-329 - I-332, Vol. 1, DOI 10.1109/ICIP.2002.1038027.

[8] F. Russo, A method for estimation and filtering of Gaussian noise in images. Instrumentation and Measurement, IEEE Transactions on Volume 52, Issue 4, Aug. 2003 pp. 1148-1154.

[9] SANG KWANG LEE, Yo-SONG Ho, Edge-preserving image restoration using adaptive constrained optimization. TENCON '98. 1998 IEEE Region 10 International Conference on Global Connectivity in Energy, Computer, Communication and Control. Vol. 1, 17-19 Dec. 1998, pp. 70-73, Vol. 1.

[10] TaO Chen, Hong ReN Wu, Space variant median filters for the restoration of impulse noise corrupted images Circuits and Systems II: Analog and Digital Signal Processing, IEEE Transactions on [see also Circuits and Systems II: Express Briefs, IEEE Transactions on] Volume 48, Issue 8, Aug. 2001, pp. 784-789. 
Contact addresses:

Leonardo Corbalán

III-LIDI (Institute of Research in Computer Sciences LIDI) Faculty of Computer Sciences

National University of La Plata

La Plata, Argentina, 1900

corbalan@lidi.info.unlp.edu.ar

Germán Osella Massa

III-LIDI (Institute of Research in Computer Sciences LIDI)

Faculty of Computer Sciences

National University of La Plata

La Plata, Argentina, 1900

gosella@lidi.info.unlp.edu.ar

Claudia Russo

III-LIDI (Institute of Research in Computer Sciences LIDI)

Faculty of Computer Sciences

National University of La Plata

La Plata, Argentina, 1900

crusso@lidi.info.unlp.edu.ar

III-LIDI (Institute of Research in Computer Sciences LIDI)

Faculty of Computer Sciences

National University of La Plata

La Plata, Argentina, 1900

laural@lidi.info.unlp.edu.ar

Armando De Giusti

III-LIDI (Institute of Research in Computer Sciences LIDI)

Faculty of Computer Sciences

National University of La Plata

La Plata, Argentina, 1900

degiusti@lidi.info.unlp.edu.ar

LEONARDO CORBALÁN has a Licentiate Degree in Computer Science, National University of La Plata, Argentina. He is currently developing his master thesis in data networks. His research interests include evolutionary algorithms, artificial neural networks, and multiagent systems.

GERMÁN OSELLA MASSA has a Licentiate Degree in Computer Science, National University of La Plata, Argentina. He is attending the $\mathrm{PhD}$ course in Computer Science, School of Computer Science (UNLP - Argentina). He currently has a Type I PhD Scholarship of the CONICET. His research interests include evolutionary algorithms, fuzzy logic and evolutionary neural networks.

Claudia Russo has a Master Degree in Computer Science. She is Co-Chair Professor at the School of Computer Science (National University of La Plata, Argentina). Her research interests include massive data treatment, in particular image processing, 3D and video reconstruction.

LAURA LANZARINI has a Licentiate Degree in Computer Science. She is currently Full Time Professor at the School of Computer Science (National University of La Plata, Argentina). Her research interests include evolutionary neural networks applied to pattern recognition, control and data mining.

ARMANDO DE GIUSTI has universitary degrees in Electronic Engineering and Computer Science. He is currently Full Time Professor at the School of Computer Science (National University of La Plata, Argentina), and Visiting Professor at different Universities. Director of the Institute of Research on Computer Sciences (III-LIDI), and CONICET Main Researcher. His research interests include concurrency, distributed and parallel processing, grid computing, real time systems, and computer technology applied to education. 